\title{
A Transaldolase From Aquilaria Sinensis Involves in Aba-mediated Seed Germination and Root Growth
}

\section{Yuqing Rong}

Beijing University of Chinese Medicine

\section{Tiezheng Li}

Beijing University of Chinese Medicine

Xiao Liu

Beijing University of Chinese Medicine

Shepo Shi

Beijing University of Chinese Medicine

Xiaohui Wang

Beijing University of Chinese Medicine

pengfei Tu ( $\square$ pengfeitu@163.com )

Beijing University of Chinese Medicine

\section{Research Article}

Keywords: Aquilaria sinensis, Transaldolase, ABA, Seed germination, Root growth

Posted Date: February 17th, 2021

DOl: https://doi.org/10.21203/rs.3.rs-234062/v1

License: (c) (1) This work is licensed under a Creative Commons Attribution 4.0 International License. Read Full License

Version of Record: A version of this preprint was published at Plant Cell, Tissue and Organ Culture (PCTOC) on July 5th, 2021. See the published version at https://doi.org/10.1007/s11240-021-02110-6. 


\section{Abstract}

Transaldolase, the key enzyme of the pentose-phosphate pathway, plays an important role in plant growth and defense. Seed germination is a key factor that influences the cultivation of Aquilaria sinensis, the plant source of agarwood, which is widely used as a traditional medicine, perfume and incenses. However, little is known about the function of transaldolase in abscisic acid (ABA)-mediated seed germination. In the present study, the full-length AsTal1 gene was isolated and characterized from $A$. sinensis calli. Sublocalization analysis indicated that AsTal1 was localized in the cytoplasm. In addition, phenotypic analysis indicated that AsTa/1-overexpressing Nicotiana benthamiana (OE) plants were less sensitive to ABA during seed germination and root growth than wild-type (WT) plants. Overexpression of AsTal1 regulated the expression of genes involved in ABA metabolism, biosynthesis and signal transduction under $A B A$ treatment. In addition, expression of $N b R b o h A$ and $N b R b o h B$ was inhibited in the overexpression lines, whereas the abundance and activities of the antioxidative enzymes SOD, APX, and POD were higher in the transgenic plants than in the WT lines after ABA treatment. Taken together, our results indicated that AsTal1 is involved in the ABA response during seed germination and root growth by regulating the expression of genes involved in the ABA signaling pathway and the enzymes responsive to ROS.

\section{Introduction}

The oxidative pentose-phosphate pathway (PPP) plays an essential role in carbohydrate metabolism, by both serving as a source of NADPH for biosynthesis and balance of reactive oxygen intermediates in plant cells and providing precursors for several main biosynthesis pathways (Hawkins et al. 2018; Yang et al. 2015). The OPPP comprises two separate branches: an oxidative branch, in which glucose-6phosphate dehydrogenase (G6PDH) and 6-phosphogluconate dehydrogenase generate ribulose 5phosphate (Ru-5-P) and NADPH, and a nonoxidative branch, in which transketolase (TK) and transaldolase (TAL) convert Ru-5-P into xylulose 5-phosphate (Xul-5-P) and ribose-5-phosphate (Rib-5-P) (Caillau and Paul Quick 2005; Hawkins et al. 2018; Stincone et al. 2015). This suggests that the main regulatory steps of the OPPP are catalyzed by TAL (Caillau and Paul Quick 2005). TAL is ubiquitous in prokaryotes and eukaryotes and was first identified as the rate-limiting enzyme in yeast (Yang et al. 2015). In humans, TAL deficiency causes pathological disorders such as hydrops fetalis, liver dysfunction, and neonatal multiorgan disease (Michel et al. 2015; Perl et al. 2011). In plants, TAL appears to be involved in plant defenses and development. For instance, TAL abundance increased in both cucumber leaves and wheat leaves upon infection by fungi (Caillau and Paul Quick 2005). The expression level of TAL in potato also increased in response to stress (Moehs et al. 1996). Yang et al. recently indicated that knockout of TAL in rice resulted in a dwarf phenotype, with narrow, short leaves and an altered vascular pattern (Yang et al. 2015). Zheng et al. reported that GSM2, a TAL from Arabidopsis thaliana, is involved in maintaining reactive oxygen species balance in response to Glc during seeding growth (Zheng et al. 2020). 
ABA is a key phytohormone that participates in the response to environmental stresses and plant growth, such as seed germination and dormancy and root growth and development (Du et al. 2019; Fujita et al. 2011; Liu et al. 2015). The promoters of ABA-mediated genes contain conserved ABA-responsive elements (ABREs) with the core nucleotide sequence ACGT, which is the binding site for basic leucine zipper (bZIP) transcription factors (Hattori et al. 2002). In Arabidopsis, the transcription factors ABI3 and $A B I 5$ play important roles in mediating the $A B A$ signaling pathway during seed germination and dormancy (Hattori et al. 2002; Kashiwakura et al. 2016). Recent studies have indicated that the expression of glucose-6-phosphate dehydrogenase (G6PDH), the other important gene in the OPPP, can be induced by $A B A$ and that G6PDH regulates seed germination through the ABA signaling pathway (Yang et al. 2019). However, the function of TAL in the ABA response is still poorly understood, despite the first TAL being identified in peas several decades ago.

Aquilaria sinensis is a tropical evergreen tree species that is distributed in Guangdong, Guangxi, Fujian and Hainan provinces in China and in other countries, including Indonesia, India, Vietnam, Malaysia and Thailand (Ding et al. 2020). It is one of the most important plant species for producing agarwood, and $A$. sinensis material has long been used as a sedative, digestive and anti-emetic drug in traditional medicine and as unique perfume and incense (Ding et al. 2020; Xu et al. 2020). Owing to the great demand and high economic value of agarwood, Aquilaria forests are facing severe destruction in nearly every country in which agarwood is commercially produced. Therefore, $A$. sinensis is listed in Appendix II of the Convention on International Trade in Endangered Species of Wild Fauna and Flora, and Aquilaria cultivation has attracted much attention in countries including China, Indonesia, India, Thailand and Malaysia (Wang et al. 2016; Xu et al. 2013). Seeding is currently the main source of cultivated $A$. sinensis sold on the market (Akhsan et al. 2015). The time of seed germination is important for seeding establishment and plant growth. Moreover, the germination ability of $A$. sinensis seeds is easily lost, and the regeneration ability is weak (Chen et al. 2016). Genetic and physiological analyses show that ABA promotes seed maturation and dormancy (Qu et al. 2020). As mentioned above, genes of the TAL family play an important role in plant resistance and plant growth, but little is known about the function of TAL family proteins in seed germination through the ABA signaling pathway. In this study, we isolated the fulllength $c D N A$ of $A s T a / 1$ and characterized the function of AsTa/1 in seed germination and root growth through the ABA pathway. AsTal1 functions by scavenging reactive oxygen species (ROS) in response to ABA. These findings improve the understanding of the roles of TAL family members in plant development and provide valuable insights for further investigation of the mechanism underlying $A$. sinensis seed germination.

\section{Materials And Methods}

\subsection{Plant materials and growth conditions}

A. sinensis calli from plant leaves were obtained as described in previous reports (Wang et al. 2016). The calli were subcultured on fresh Murashige and Skoog (MS) media comprising $1 \mu \mathrm{g} / \mathrm{mL}$ 6-benzyladenin (6BA), $2 \mu \mathrm{g} / \mathrm{mL}$ naphthylacetic acid (NAA), $1 \mu \mathrm{g} / \mathrm{mg}$ kinetin (KT), and $1 \mu \mathrm{g} / \mathrm{mL}$ dichlorophenoxyacetic acid 
(2,4-D) for 15 days and then cultured on media supplemented with $100 \mu \mathrm{M} \mathrm{ABA}, 200 \mathrm{mM} \mathrm{NaCl}, 750 \mathrm{mM}$ mannitol, or $500 \mu \mathrm{M} \mathrm{CdCl}_{2}$. For low-temperature treatment, the calli were cultured in a $4^{\circ} \mathrm{C}$ environment. Calli from different treatment groups and the control group were harvested at different time points $(0 \mathrm{~h}$, $12 \mathrm{~h}, 24 \mathrm{~h}, 36 \mathrm{~h}, 48 \mathrm{~h}$ ). Transgenic and wild-type $N$. benthamiana seeds were surface sterilized for $30 \mathrm{~s}$ in $75 \%$ ethanol, sterilized with $2 \% \mathrm{NaClO}$ for 10 min, rinsed with sterile water four times, and then cultivated in 1/2-strength MS media supplemented with different concentrations of ABA in a growth chamber at $25^{\circ} \mathrm{C}$ (14 h light/10 $\mathrm{h}$ dark).

\subsection{RNA preparation and cDNA for AsTal1 analysis}

Total RNA was isolated from the samples of $A$. sinensis calli using Total RNA Purification Reagent (Norgen, Cat\# 17200) according to the manufacturer's instructions. The quantity and integrity of the RNA samples were determined by a NanoDrop ND-2000 spectrophotometer and 1.2\% agarose electrophoresis. Reverse-transcription reactions were performed with $1 \mu \mathrm{g}$ of total RNA, $200 \mathrm{U}$ of M-MLV transcriptase (Promega, Madison, WI, USA), dNTPs ( $0.2 \mathrm{mM}$ each), $20 \mathrm{U}$ of RNase inhibitor (Invitrogen) and $0.1 \mu \mathrm{M}$ oligo dT (18) primer in a final volume of $30 \mu \mathrm{L}$. Reverse transcription was carried out at $42^{\circ} \mathrm{C}$ for 90 min. The AsTal1 cDNA sequence containing class-specific TAL conserved domains and nucleotide sequences was obtained from the $A$. sinensis callus transcriptomic dataset. To isolate this gene from $A$. sinensis calli, open reading frames (ORFs) were isolated via RT-PCR using specific primers (Table 1). The resulting DNA fragments were ligated into a pMD19-T vector, and three clones were sequenced in both directions. The ORFs of the CDNA sequence of AsTAL 1 were predicted with the ORF finder online tool (http://www.ncbi.nlm.nih.gov/projects/gorf/). The amino acid alignments were performed by DNAMAN software, and phylogenetic trees were constructed using the neighbor-joining tree algorithm of the MEGA 6.0 program.

\subsection{Subcellular localization}

The ORF of AsTal1 was isolated by PCR amplification using specific primers (Table S1). The fragments were fused to the C-terminus of the green fluorescent protein (GFP) gene in a pCAMBIA1300-35S-EGFP binary vector. The binary vectors were then transformed into Agrobacterium tumefaciens strain EHA105, after which the transformed Agrobacterium were infiltrated into Nicotiana benthamiana leaves as described in previous reports. Subcellular localization was observed via laser confocal scanning microscopy.

\subsection{Quantitative real-time PCR (qRT-PCR) assays}

qRT-PCR was performed on a CFX Connect ${ }^{\mathrm{TM}}$ Real-time System (Bio-Rad) using TransStart Tip Green qPCR SuperMix (Transgen) according to the manufacturer's protocol. The $A$. sinensisGAPDH gene and the $N$. benthamiana $\beta$-actin gene were used as internal controls to normalize RNA levels. Real-time PCR was initiated with $30 \mathrm{~s}$ of incubation at $94^{\circ} \mathrm{C}$, followed by 40 cycles of $94^{\circ} \mathrm{C}$ for $5 \mathrm{~s}$ and $60^{\circ} \mathrm{C}$ for $30 \mathrm{~s}$. The primers used for qRT-PCR were generated by the Primer 5.0 program (Table S1), and the $2^{-\triangle \triangle C T}$ method was used to analyze the expression level of AsTal1 genes. Three biological experiments and three 
experimental replicates were included to analyze gene expression. The primers used for qRT-PCR are listed in Table S1.

\section{$2.5 N$. benthamiana transformation}

To construct AsTal1 overexpression vectors, the AsTal1 ORF was obtained by specific primers and subsequently subcloned into a pCAMBIA1300-35S binary vector to yield pCAMBIA1300-35S-AsTal1 vectors. The pCAMBIA1300-35S-AsTal1 plasmids were then transformed into wild-type $N$. benthamiana using the Agrobacterium-mediated leaf disc transformation method. Positive transgenic $N$. benthamiana plants were identified through PCR and qPCR. T3 seeds of three independent overexpression lines (OE) and wild type (WT) plants were used to analyze the function of the AsTal1 gene.

\subsection{Analysis of ROS}

The concentration of total protein extracted from the roots of OE and WT lines was quantified with the BCA method. The $\mathrm{H}_{2} \mathrm{O}_{2}$ and $\mathrm{O}_{2}{ }^{-}$contents and the activities of antioxidant enzymes such as peroxidase (APX), superoxide dismutase (SOD), and peroxidase (POD) were measured using detection kits (Nanjing Jianchen Bioengineering Institute, China) according to the manufacturer's protocols.

\section{Results}

\subsection{Identification and characterization of AsTal1}

The AsTal1 cDNA sequence had an ORF of 1203 bp, encoding polypeptides 401 residues in length, with a calculated MW of $43.32 \mathrm{kDa}$. A previous database showed that TAL is putatively encoded by two distinct genes in Arabidopsis (AtTal1 and AtTal2). Multiple alignments indicated that the sequence of AsTal1 was $72.35 \%$ identical to that of AtTal1, sharing a homology of $17.89 \%$ with AtTal2, suggesting that the amino acid sequence of AsTal1 was more similar to AtTal1 than AtTal2. As shown in Fig. 1A, AsTal1 contained a highly conserved site (KIPAT) (Caillau and Paul Quick 2005). A phylogenetic tree was constructed from a ClustalX multiple amino acid sequence alignment of plant TALs. The results indicated that TAL family proteins from plants existed as two putative isoforms (TAL types I and II) and that AsTal1 was classified as TAL type I (Fig. 1B) (Caillau and Paul Quick 2005).

\subsection{Subcellular localization of AsTal1}

To determine the subcellular localization of AsTal1, the coding sequence of AsTal1 was cloned into a pCAMBIA1300-35S-EGFP vector. The recombinant vectors pCAMBIA1300-35S-EGFP-AsTal1 and pCAMBIA1300-35S-EGFP were subsequently transformed into $N$. benthamiana epidermal cells by the agroinfiltration method. After two or three days of incubation, the expression of the introduced gene was examined via laser confocal scanning microscopy. Confocal microscopy of the $N$. benthamiana epidermal cells of the infiltrated leaves indicated that GFP:AsTal1 was localized in the cytoplasm (Fig. 2A), whereas the control GFP was distributed across the whole epidermal cell (Fig. 2B). 


\subsection{Expression profiles of AsTal1 in different tissues and in response to abiotic stresses}

To determine the expression of AsTal1 in different tissues, relative quantitative real-time PCR analysis was performed on the total RNA from the roots, stems, leaves and shoot tips (Fig. 3A). The results showed that AsTal1 was expressed in all of the tested tissues, with tissue-specific expression patterns. AsTal1 expression was shown to be highest in the shoot tips, followed by the roots and stems. To investigate the putative role of AsTal1, the transcript levels after exposure to ABA, salt, drought, cold temperature and heavy metal stress were measured. As shown in Fig. 3B-3F, the expression of AsTal1 was slightly induced by drought, cold temperature, salt stress and heavy metal stress, while the transcript level of AsTa/1 was markedly induced by ABA treatment.

3.4 Overexpression of AsTal1 enhances the seed germination and root length of $N$. benthamiana under ABA treatment

Transgenic N. benthamiana plants overexpressing AsTal1 were generated to investigate the functions of the AsTal1 gene. Three homozygous transgenic lines were evaluated via qRT-PCR and selected for functional analysis. To elucidate whether ABA sensitivity was influenced by AsTal1, seeds of OE and WT $N$. benthamiana were germinated on 1/2-strength MS media supplemented with different concentrations of ABA. As shown in Fig. 4A-4B, the germination rates of the OE and WT lines were similar under control conditions. Seed germination was significantly inhibited for both OE and WT lines in the presence of 1 and $2 \mu \mathrm{M} \mathrm{ABA}$, but the suppression of OE plant germination was much weaker than that of the WT. For instance, the germination of OE lines reached $85.5 \%$ compared with $64.0 \%$ for WT line after 5 days of treatment with $1 \mu \mathrm{M} \mathrm{ABA}$, while $62.6 \%$ of the OE seeds compared with $36.7 \%$ of the WT seeds after 5 days of treatment with $2 \mu \mathrm{M}$ ABA (Fig. 4B). To further confirm the transgenic phenotypes, the primary root length was measured. Under normal growth conditions, no significant difference in primary root growth was detected between the WT and three OE lines (Fig. 4C-4D). However, the primary root growth of the OE plants was longer than that of the WT lines after treatment with $10 \mu \mathrm{M}$ ABA (Fig. 4D), indicating that the OE lines were hyposensitive to ABA treatment.

\subsection{AsTal1 affects genes involved in the ABA signaling pathway}

To determine the function of AsTal1 in the ABA signaling pathway, we examined the expression of ABA signaling-related genes, including those involved in $A B A$ biosynthesis, catabolism and signal transduction. The qRT-PCR results showed that the expression level of genes involved in ABA biosynthesis (NCED) was lower in the OE lines than in the WT plants under ABA treatment (Fig. 5A). In contrast, the catabolism-related genes CYP707A1 and CYP707A2 presented higher expression levels in the $O E$ lines than in the WT under ABA treatment (Fig. 5B-5C). Furthermore, our results indicated that the transcript levels of the $A B A$ signaling-related transcription factors $A B / 3$ and $A B / 5$ significantly decreased 
in the OE lines after ABA treatment (Fig. 5D-5E). These results suggested that AsTal1 is involved in the regulation of ABA metabolism and signal transduction.

\subsection{Overexpression of AsTal1 reduces ROS levels under ABA treatment}

A previous investigation showed that ABA causes ROS production and oxidative damage. To further determine the effects of AsTal1 on ABA responses, ROS accumulation was evaluated. Our results indicated that the $\mathrm{H}_{2} \mathrm{O}_{2}$ content in the OE lines was less than that in WT lines and that the $\mathrm{O}_{2}{ }^{-} \mathrm{Content}^{-}$ significantly decreased in the OE lines (Fig. 6A-6B). In plants, ROS can be generated by amino oxidases, oxygen photoreduction and peroxidases. In plants, the main ROS are produced by NADPH oxidases, which play an important role in primary root growth under different stresses. To determine whether the function of $A s T a l 1$ in response to ABA is involved in the NADPH oxidase pathway, we analyzed the expression of NADPH oxidase genes in OE and WT lines treated with ABA. As shown in Fig. 6C-6D, the expression levels of $N b R b o h A$ and $N b R b o h B$ significantly increased after ABA treatment in the WT and OE lines. However, the $N b R b o h A$ and $N b R b o h B$ expression levels in the OE lines were markedly lower than those in the WT line under ABA treatment. These results suggested that AsTal1 is involved in NADPH oxidase-dependent ROS production; antioxidant enzymes remove extra ROS to maintain the balance between ROS production and scavenging under different environmental stresses. Furthermore, we measured the expression and activity of antioxidant enzymes in OE and WT lines with or without ABA treatment. The results indicated that ABA-induced transcript levels and activity of SOD, APX and POD in the OE lines were significantly higher than those in the WT plants (Fig. 6E-6I). These results indicate that AsTal1 could enhance the capacity to scavenge excess ROS by regulating the expression and activity of antioxidant enzymes.

\section{Discussion}

A. sinensis is the main plant species that produced agarwood used as traditional medicine, incense and perfume (Ding et al. 2020). Owing to the economic value and endangered nature of $A$. sinensis plants, cultivation of $A$. sinensis is urgently needed. Seed germination is the vital factor that influences the cultivation of $A$. sinensis (Chen et al. 2016). Recent research has demonstrated that TALs also play vital roles in plant development. Here, we isolated a TAL gene, AsTal1, which belongs to the type I TAL family. Further experiments revealed that AsTal1 is involved in the response to ABA during seed germination and root development. Compared with WT plants, AsTa/1-overexpressing lines exhibited a higher seed germination rate and longer primary roots under ABA treatment.

Previous studies have shown that the expression or protein content of TALs could be induced after exposure to biotic stresses. For instance, the expression level of the $T A L$ gene in cucumber leaves and the protein content of ToTal1 in potato were significantly induced by fungal infection (Caillau and Paul Quick 2005; Moehs et al. 1996). In this investigation, our results revealed that the transcript level of AsTal1 was 
induced by abiotic stresses such as salt, drought, cold and heavy metal stress. Furthermore, the expression level of AsTal1 was significantly induced by ABA treatment, suggesting that AsTa/1 functions in ABA signaling.

$A B A$ is an important phytohormone that regulates seed dormancy and germination and root development (Chen et al. 2014; Zhong et al. 2015). G6PDH, the other important enzyme of the OPPP, is involved in ABAinducible repressors of seed germination in the ABA response (Du et al. 2019). Our results first showed that AsTal1 is also involved in the response to ABA during seed germination and root growth. Previous investigations indicated that overexpression of AtNCED3 and LeNCED1 enhances endogenous ABA accumulation (Espasandin et al. 2014; Zhang et al. 2009). Cytochrome P450 (CYP) 707A family proteins, the key enzymes involved in ABA catabolism, regulate ABA levels in plant cells (Kushiro et al. 2004; Todoroki and Ueno 2010). Our results indicated that overexpression of AsTal1 could suppress the expression level of NbNCED and increase the transcript levels of NbCYP707A1 and NbCYP707A2, suggesting that $A s T a / 1$ is involved in the $A B A$ response by regulating genes involved in $A B A$ biosynthesis and metabolism. The transcription factors $A B I 3$ and $A B I 5$ play vital roles in mediating $A B A$ signaling during seed germination and dormancy (Bi et al. 2017; Monke et al. 2004; Pan et al. 2018). The transcript levels of $A B / 3$ and $A B I 5$ were inhibited in the AsTal1-overexpressing lines under $A B A$ treatment in the present study, suggesting that the seed germination defects in the AsTa/1-overexpressing lines are mediated by ABA-responsive transcription factors.

Superoxide anion $\left(\mathrm{O}_{2}^{-}\right)$, hydroxyl radical $\left(\mathrm{HO}^{-}\right)$and hydrogen peroxide $\left(\mathrm{H}_{2} \mathrm{O}_{2}\right)$ radicals are produced at low concentrations in plant cells during plant growth and development (Mhamdi and Van Breusegem 2018; Zhou et al. 2020). It has been reported that exogenous ABA can induce ROS production in plant cells (Yan et al. 2007). Thus, we measured the influence of oxidative levels in the seeds and roots of AsTal1 OE lines and WT lines under ABA treatment. Our results showed that the ROS content was lower in the OE lines than in the WT lines under ABA treatment. ROS produce NADPH oxidase, which plays a vital role in the ABA response in maize and in ABA-inhibited primary root growth in Arabidopsis (Kwak et al. 2003). Our investigation indicated that overexpressing AsTa/1 inhibited the expression levels of NbrbohA and NbrbohB. Due to the physicochemical toxicity of ROS, plants have a set of antioxidant enzymes, such as APX, SOD and POD, to scavenge excess ROS and maintain the balance between ROS production and scavenging (ljaz et al. 2017; Xu et al. 2018). Therefore, we also measured the abundance and activities of antioxidant enzymes such as APX, SOD, and POD in AsTa/1 OE lines and WT lines under ABA treatment. The results suggested that AsTal1 overexpression could enhance antioxidant enzyme activities and increase expression levels to remove excess ROS under ABA treatment.

\section{Abbreviations}

ABA: abscisic acid; NAA: naphtha acetic acid; 6-BA: 6-benzyladenim; 2,4-D: dichlorophenoxyacetic acid; KT: kinetin; TAL: Transaldolase

\section{Declarations}


Acknowledgements

This research was supported by Science Foundation of Beijing University of Chinese Medicine (2019-JYBJS-014)

\section{Author contributions}

YQR and TZL carried out the experiments and wrote the draft of the paper. $X L$ and SPS participated in the preparation of the manuscript. XHW and PFT designed the research and supervised the work throughout. All the authors read and approved the final manuscript.

\section{Compliance with ethical standards}

\section{Conflict of interest}

The authors declare that they have no conflict of interest.

\section{References}

1. Akhsan N, Mardji D, Sutisna M (2015) Response of Aquilaria Microcarpa to Two Species of Fusarium under Two Different Cultivation Systems. Journal of Tropical Forest Science 27: 447-455

2. Bi C, Ma Y, Wu Z, Yu YT, Liang S, Lu K, Wang XF (2017) Arabidopsis ABI5 plays a role in regulating ROS homeostasis by activating CATALASE 1 transcription in seed germination. Plant Mol Biol 94: 197-213

3. Caillau M, Paul Quick W (2005) New insights into plant transaldolase. Plant J 43: 1-16

4. Chen C, Letnik I, Hacham Y, Dobrev P, Ben-Daniel BH, Vankova R, Amir R, Miller G (2014) ASCORBATE PEROXIDASE6 protects Arabidopsis desiccating and germinating seeds from stress and mediates cross talk between reactive oxygen species, abscisic acid, and auxin. Plant Physiol 166: 370-383

5. Chen G, Liu C, Sun W (2016) Pollination and seed dispersal of Aquilaria sinensis (Lour.) Gilg (Thymelaeaceae): An economic plant species with extremely small populations in China. Plant Divers 38: 227-232

6. Ding X, Mei W, Lin Q, Wang H, Wang J, Peng S, Li H, Zhu J, Li W, Wang P, Chen H, Dong W, Guo D, Cai C, Huang S, Cui P, Dai H (2020) Genome sequence of the agarwood tree Aquilaria sinensis (Lour.) Spreng: the first chromosome-level draft genome in the Thymelaeceae family. Gigascience 9

7. Du HY, Chen GS, Yu JM, Bao YY, Liu GT, Liu HP, Gupta R (2019) Involvement of putrescine in osmotic stress-induced ABA signaling in leaves of wheat seedlings. J Biosci 44

8. Espasandin FD, Maiale SJ, Calzadilla P, Ruiz OA, Sansberro PA (2014) Transcriptional regulation of 9cis-epoxycarotenoid dioxygenase (NCED) gene by putrescine accumulation positively modulates ABA synthesis and drought tolerance in Lotus tenuis plants. Plant Physiol Biochem 76: 29-35

9. Fujita Y, Fujita M, Shinozaki K, Yamaguchi-Shinozaki K (2011) ABA-mediated transcriptional regulation in response to osmotic stress in plants. J Plant Res 124: 509-525 
10. Hattori T, Totsuka M, Hobo T, Kagaya Y, Yamamoto-Toyoda A (2002) Experimentally determined sequence requirement of ACGT-containing abscisic acid response element. Plant Cell Physiol 43: $136-140$

11. Hawkins JP, Ordonez PA, Oresnik IJ (2018) Characterization of Mutations That Affect the Nonoxidative Pentose Phosphate Pathway in Sinorhizobium meliloti. J Bacteriol 200

12. Ijaz R, Ejaz J, Gao S, Liu T, Imtiaz M, Ye Z, Wang T (2017) Overexpression of annexin gene AnnSp2, enhances drought and salt tolerance through modulation of $A B A$ synthesis and scavenging ROS in tomato. Sci Rep 7: 12087

13. Kashiwakura Y, Kobayashi D, Jikumaru Y, Takebayashi Y, Nambara E, Seo M, Kamiya Y, Kushiro T, Kawakami N (2016) Highly Sprouting-Tolerant Wheat Grain Exhibits Extreme Dormancy and Cold Imbibition-Resistant Accumulation of Abscisic Acid. Plant Cell Physiol 57: 715-732

14. Kushiro T, Okamoto M, Nakabayashi K, Yamagishi K, Kitamura S, Asami T, Hirai N, Koshiba T, Kamiya Y, Nambara E (2004) The Arabidopsis cytochrome P450 CYP707A encodes ABA 8'-hydroxylases: key enzymes in ABA catabolism. EMBO J 23: 1647-1656

15. Kwak JM, Mori IC, Pei ZM, Leonhardt N, Torres MA, Dangl JL, Bloom RE, Bodde S, Jones JD, Schroeder JI (2003) NADPH oxidase AtrbohD and AtrbohF genes function in ROS-dependent ABA signaling in Arabidopsis. EMBO J 22: 2623-2633

16. Liu R, Liu Y, Ye N, Zhu G, Chen M, Jia L, Xia Y, Shi L, Jia W, Zhang J (2015) AtDsPTP1 acts as a negative regulator in osmotic stress signalling during Arabidopsis seed germination and seedling establishment. J Exp Bot 66: 1339-1353

17. Mhamdi A, Van Breusegem F (2018) Reactive oxygen species in plant development. Development 145

18. Michel S, Keller MA, Wamelink MM, Ralser M (2015) A haploproficient interaction of the transaldolase paralogue NQM1 with the transcription factor VHR1 affects stationary phase survival and oxidative stress resistance. BMC Genet 16: 13

19. Moehs CP, Allen PV, Friedman M, Belknap WR (1996) Cloning and expression of transaldolase from potato. Plant Mol Biol 32: 447-452

20. Monke G, Altschmied L, Tewes A, Reidt W, Mock HP, Baumlein H, Conrad U (2004) Seed-specific transcription factors ABI3 and FUS3: molecular interaction with DNA. Planta 219: 158-166

21. Pan J, Wang H, Hu Y, Yu D (2018) Arabidopsis VQ18 and VQ26 proteins interact with ABI5 transcription factor to negatively modulate ABA response during seed germination. Plant J 95: 529544

22. Perl A, Hanczko R, Telarico T, Oaks Z, Landas S (2011) Oxidative stress, inflammation and carcinogenesis are controlled through the pentose phosphate pathway by transaldolase. Trends Mol Med 17: 395-403

23. Qu L, Sun M, Li X, He R, Zhong M, Luo D, Liu X, Zhao X (2020) The Arabidopsis F-box protein FOF2 regulates ABA-mediated seed germination and drought tolerance. Plant Sci 301: 110643 
24. Stincone A, Prigione A, Cramer T, Wamelink MM, Campbell K, Cheung E, Olin-Sandoval V, Gruning NM, Kruger A, Tauqeer Alam M, Keller MA, Breitenbach M, Brindle KM, Rabinowitz JD, Ralser M (2015) The return of metabolism: biochemistry and physiology of the pentose phosphate pathway. Biol Rev Camb Philos Soc 90: 927-963

25. Todoroki Y, Ueno K (2010) Development of specific inhibitors of CYP707A, a key enzyme in the catabolism of abscisic acid. Curr Med Chem 17: 3230-3244

26. Wang X, Gao B, Liu X, Dong X, Zhang Z, Fan H, Zhang L, Wang J, Shi S, Tu P (2016) Salinity stress induces the production of 2-(2-phenylethyl)chromones and regulates novel classes of responsive genes involved in signal transduction in Aquilaria sinensis calli. BMC Plant Biol 16: 119

27. Xu N, Chu Y, Chen H, Li X, Wu Q, Jin L, Wang G, Huang J (2018) Rice transcription factor OsMADS25 modulates root growth and confers salinity tolerance via the ABA-mediated regulatory pathway and ROS scavenging. PLoS Genet 14: e1007662

28. Xu Y, Zhang Z, Wang M, Wei J, Chen H, Gao Z, Sui C, Luo H, Zhang X, Yang Y, Meng H, Li W (2013) Identification of genes related to agarwood formation: transcriptome analysis of healthy and wounded tissues of Aquilaria sinensis. BMC Genomics 14: 227

29. Xu YH, Sun PW, Tang XL, Gao ZH, Zhang Z, Wei JH (2020) Genome-wide analysis of WRKY transcription factors in Aquilaria sinensis (Lour.) Gilg. Sci Rep 10: 3018

30. Yan J, Tsuichihara N, Etoh T, Iwai S (2007) Reactive oxygen species and nitric oxide are involved in ABA inhibition of stomatal opening. Plant Cell Environ 30: 1320-1325

31. Yang L, Wang S, Sun L, Ruan M, Li S, He R, Zhang W, Liang C, Wang X, Bi Y (2019) Involvement of G6PD5 in ABA response during seed germination and root growth in Arabidopsis. BMC Plant Biol 19: 44

32. Yang Z, Zhou Y, Huang J, Hu Y, Zhang E, Xie Z, Ma S, Gao Y, Song S, Xu C, Liang G (2015) Ancient horizontal transfer of transaldolase-like protein gene and its role in plant vascular development. New Phytol 206: 807-816

33. Zhang M, Leng P, Zhang G, Li X (2009) Cloning and functional analysis of 9-cis-epoxycarotenoid dioxygenase (NCED) genes encoding a key enzyme during abscisic acid biosynthesis from peach and grape fruits. J Plant Physiol 166: 1241-1252

34. Zheng M, Zhu C, Yang T, Qian J, Hsu YF (2020) GSM2, a transaldolase, contributes to reactive oxygen species homeostasis in Arabidopsis. Plant Mol Biol 104: 39-53

35. Zhong C, Xu H, Ye S, Wang S, Li L, Zhang S, Wang X (2015) Gibberellic Acid-Stimulated Arabidopsis6 Serves as an Integrator of Gibberellin, Abscisic Acid, and Glucose Signaling during Seed Germination in Arabidopsis. Plant Physiol 169: 2288-2303

36. Zhou X, Xiang Y, Li C, Yu G (2020) Modulatory Role of Reactive Oxygen Species in Root Development in Model Plant of Arabidopsis thaliana. Front Plant Sci 11: 485932

\section{Figures}




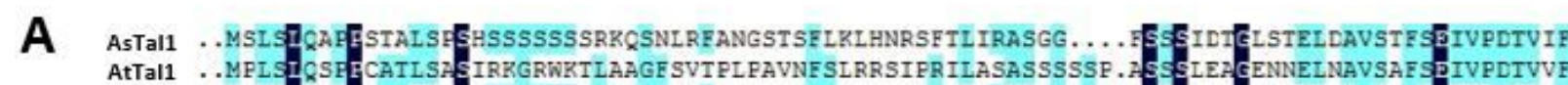

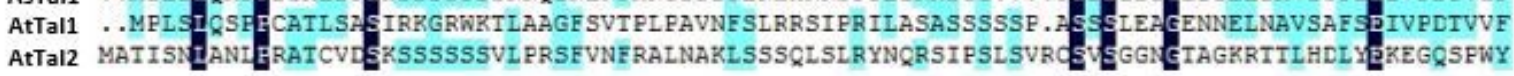

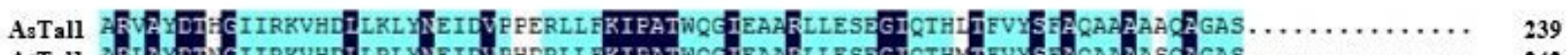

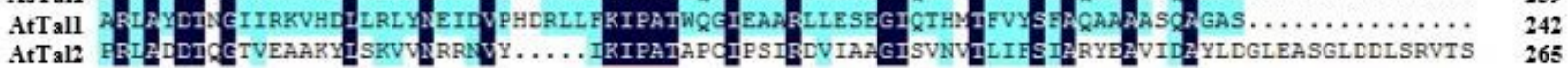

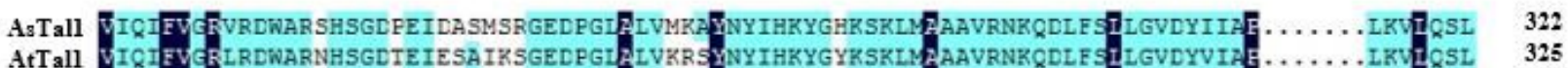

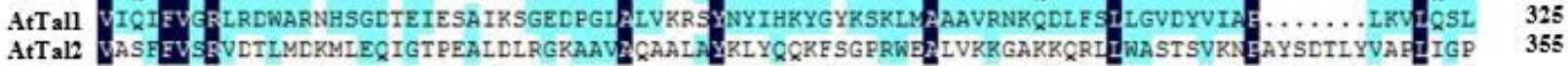

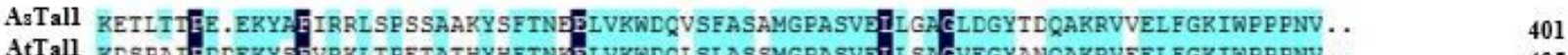

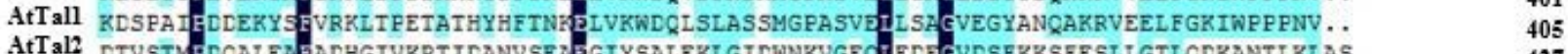

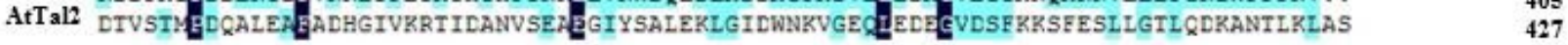

B

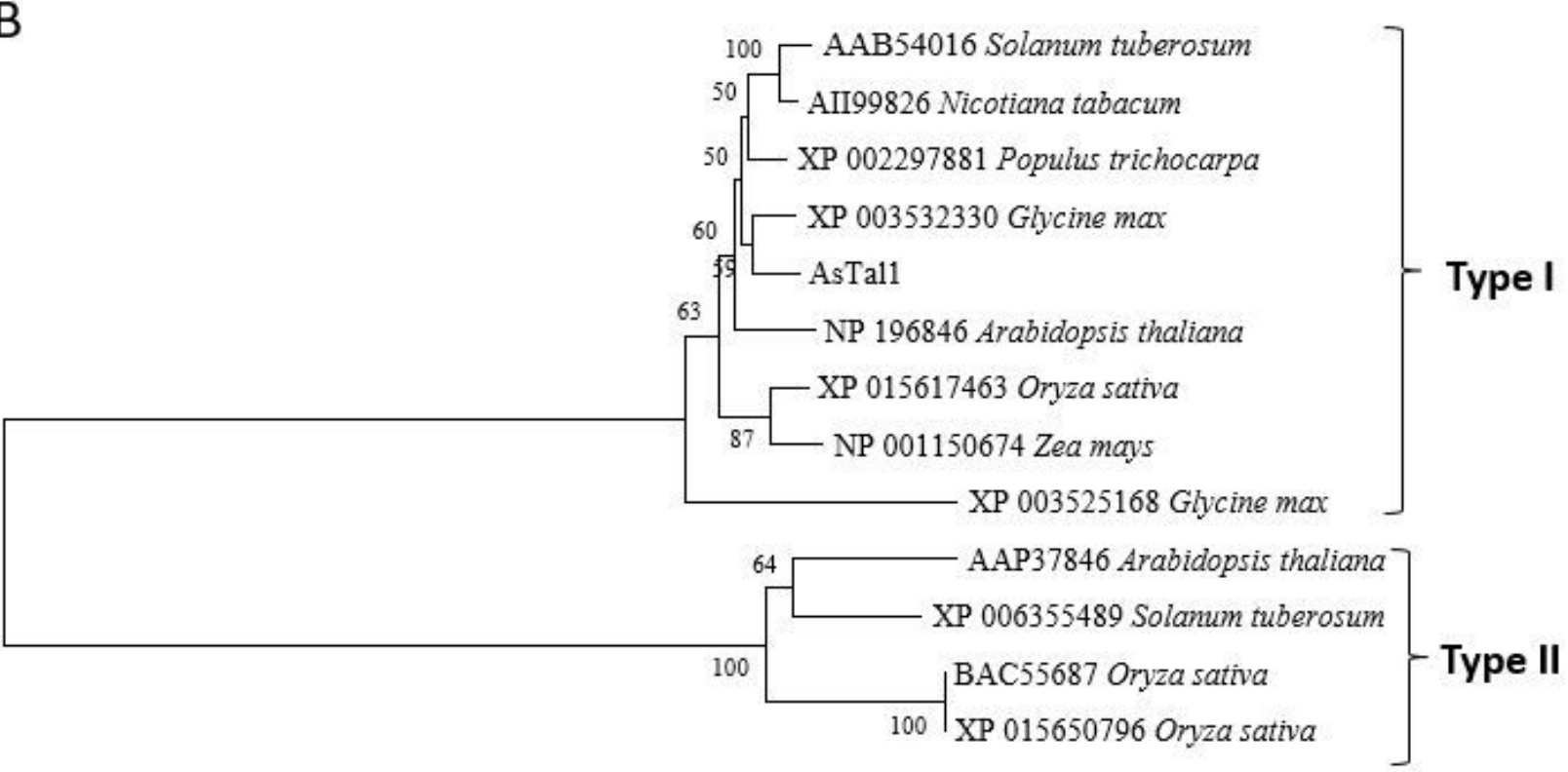

\section{Figure 1}

AsTal1 sequence analysis. (A) Multiple sequence alignment of AsTal1 with AtTal1 and AtTal2. Red line indicates conserved site. (B) Phylogenetic analysis of AsTal1 and the TAL protein from other plants. Multiple sequence alignments of TAL sequences were performed using ClustalX, and the phylogenetic tree was constructed using MEGA6 with the neigbor-joining ( $\mathrm{NJ}$ ) method and 1000 bootstrap. The TAL proteins were classified into two groups (I, II). 
A

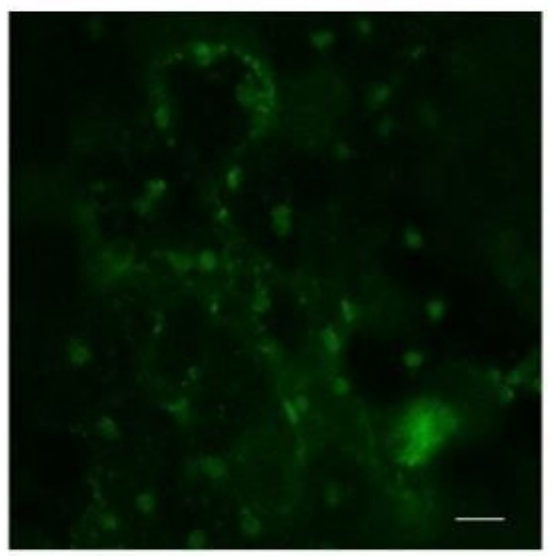

B

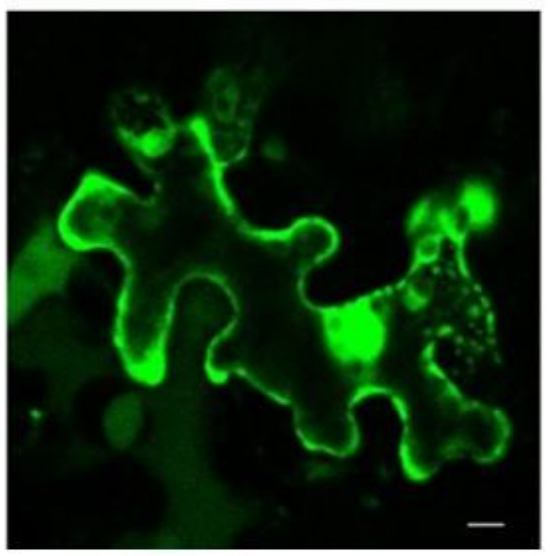

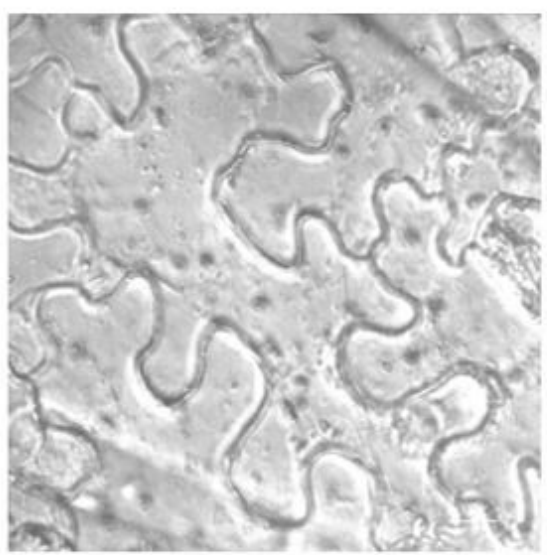
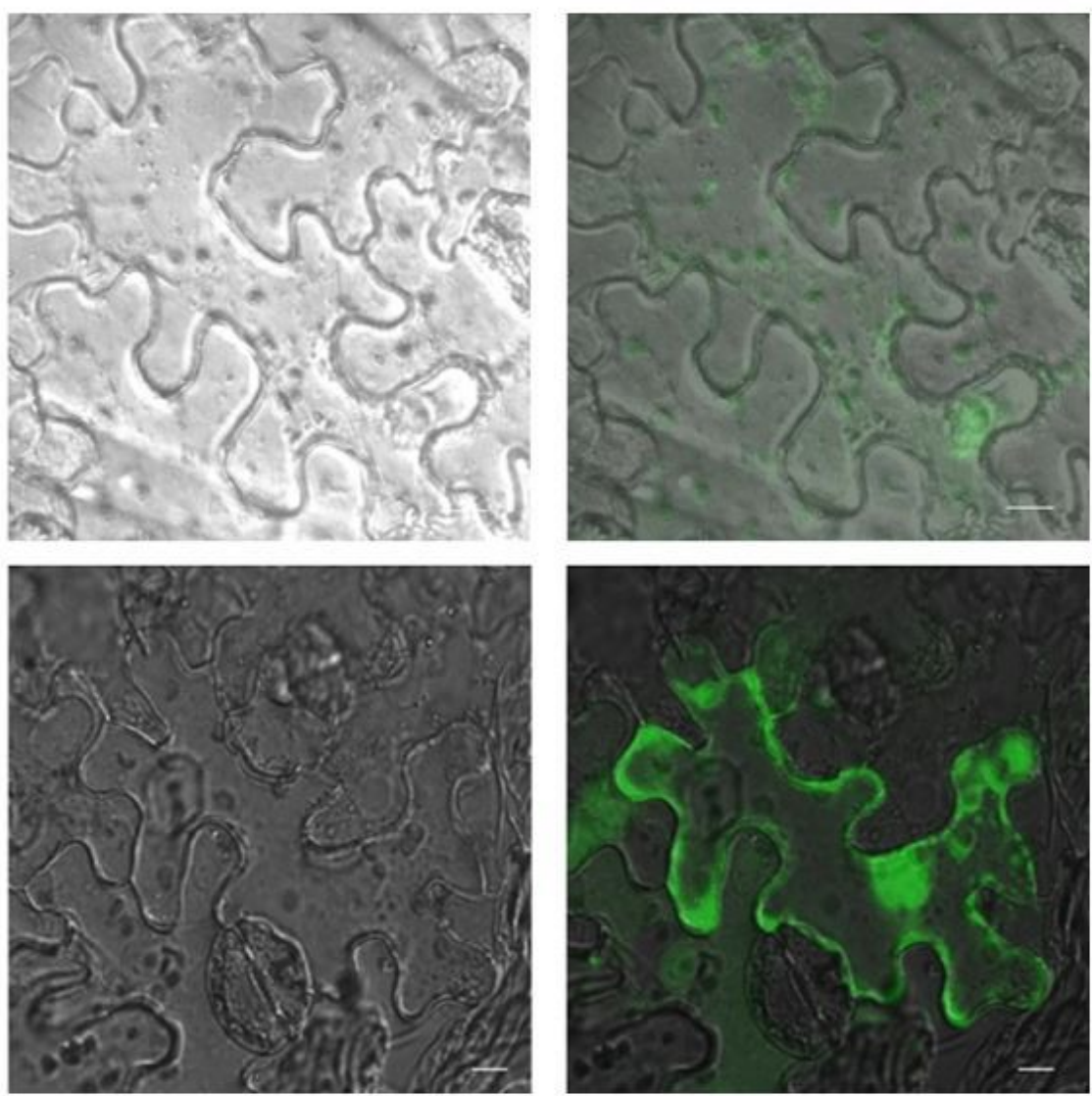

\section{Figure 2}

Subcellular localization of AsTal1 in epidermal cells of N.benthamiana leaves. (A) Epidermal leaf cells agroinfiltrated with pCAMBIA1300-35S-EGFP-AsTal1; (B) Epidermal cells agroinfiltrated with pCAMBIA1300-35S-EGFP for transient expression. Bars $=10 \mu \mathrm{m}$. 
A
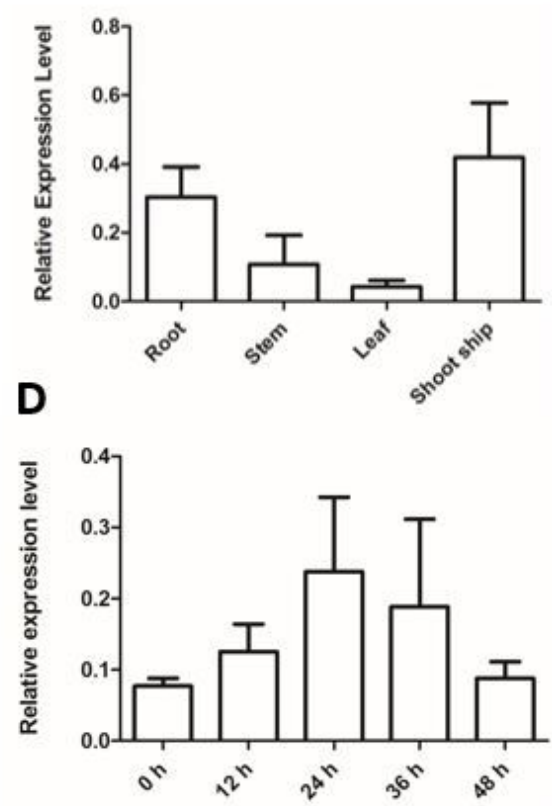

B

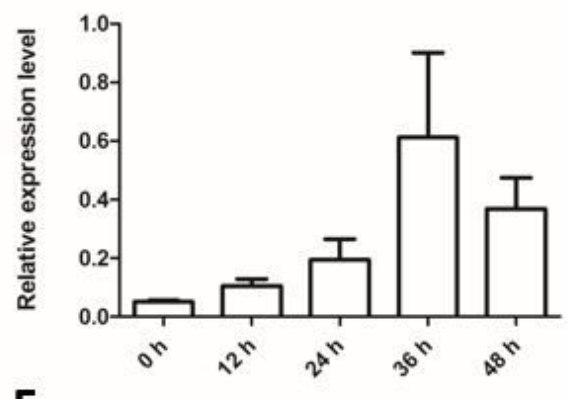

E

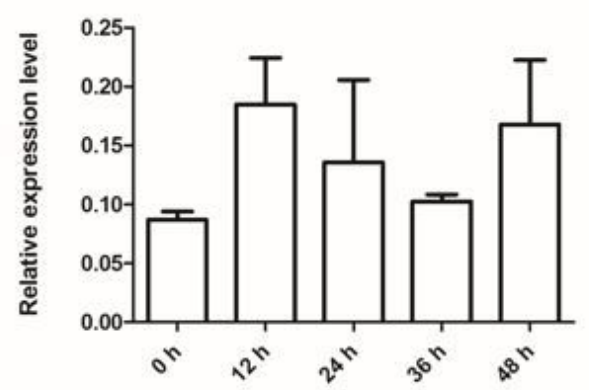

C
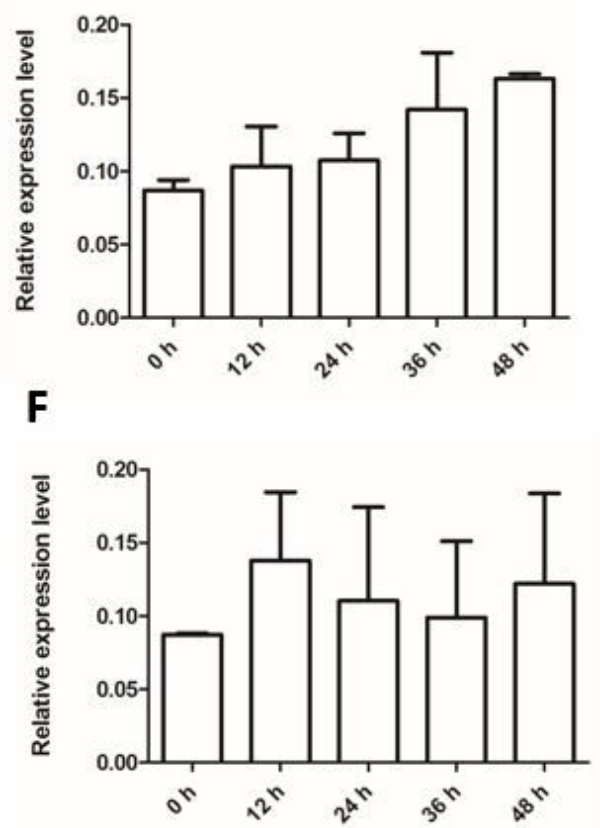

Figure 3

Expression profiles of AsTal1 in different tissues and under adverse stress treatments. qRT-PCR was performed with the total RNA which extracted from different tissues and calli treated with $A B A, N a C l$, mannitol, cold and $\mathrm{CdCl} 2$. (A) Expression level of AsTal1 in different tissues; (B)-(F) Expression level of AsTal1 under $\mathrm{ABA}, \mathrm{NaCl}$, mannitol, cold and $\mathrm{CdCl} 2$, respectively. The GAPDH gene was used as an internal control, and the experiment was repeated with at least three times. Values are means standard error $(n \geq 3)$. Statistical significance was determined using Student's t-test $\left(* P<0.05 ;{ }^{*} P<0.01\right)$. 
A
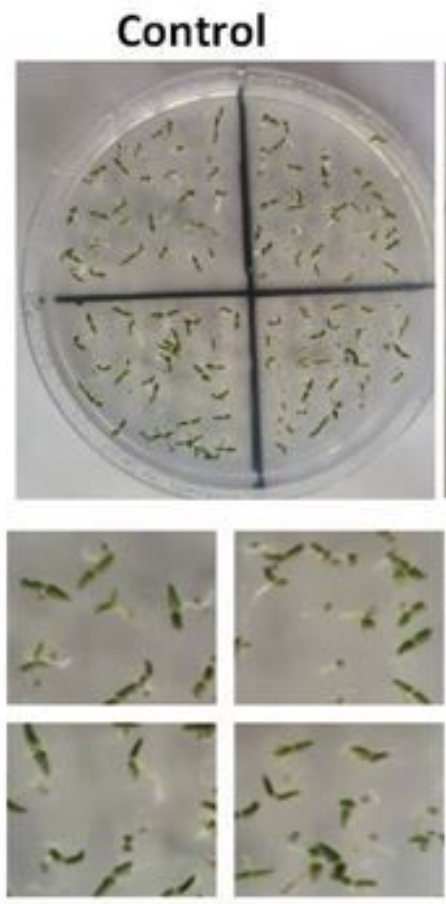

B
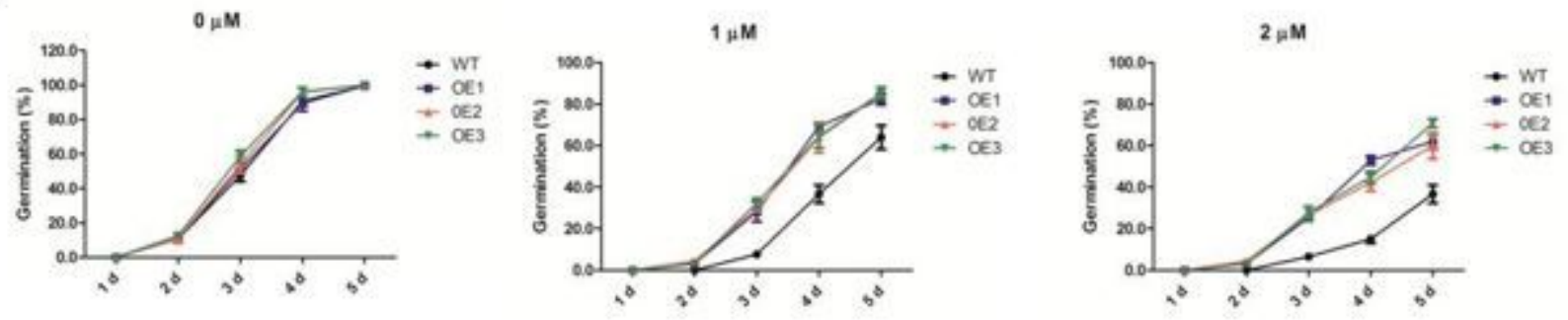

C

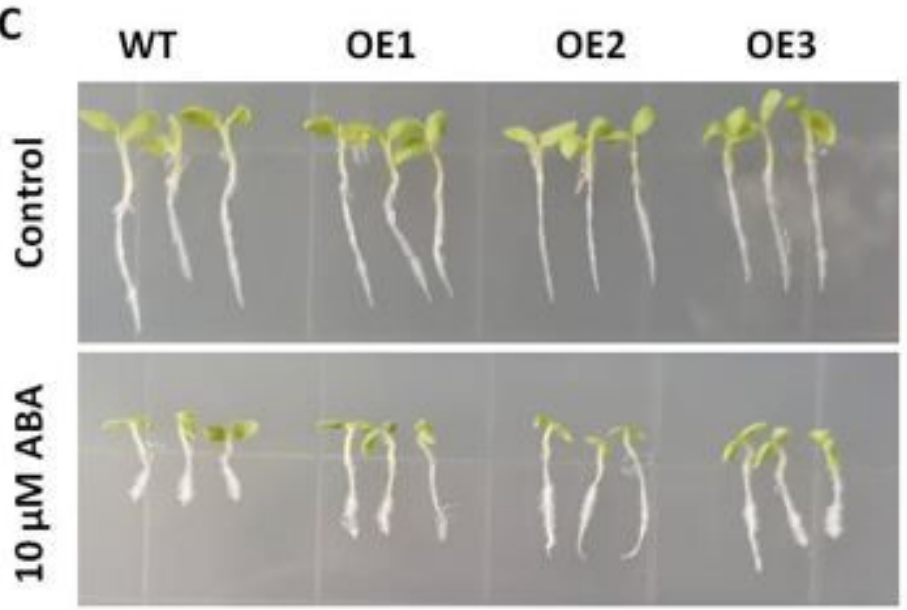

D

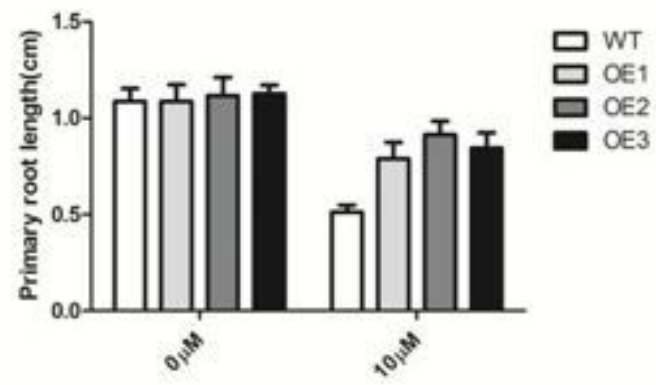

\section{Figure 4}

Seed germination and root growth of wild type and AsTal1 overexpression lines in response to ABA. (A) Seeds were germinated on $1 / 2 \mathrm{MS}$ plates with or without ABA. Photographs were taken at 5 day under ABA treatment. (B) Seed germination percentage with or without ABA treatment at different day. (C) Fourday-old seedings were cultured vertically on $1 / 2 \mathrm{MS}$ medium supplied with $10 \mu \mathrm{M}$ ABA for 4 days. (D) The root length of WT and OE lines with or without ABA treatment at 4 day. 
A

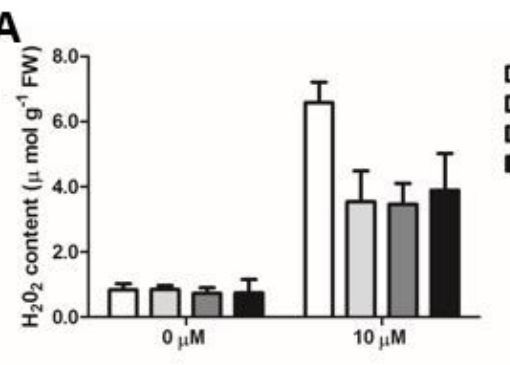

D

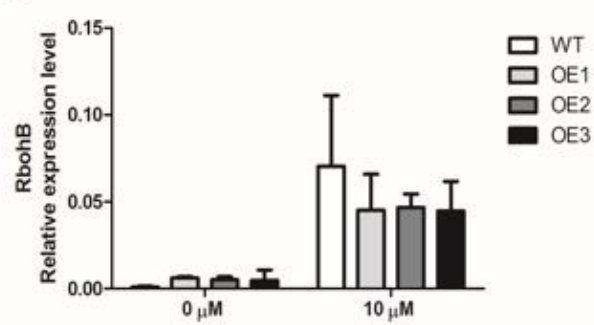

G

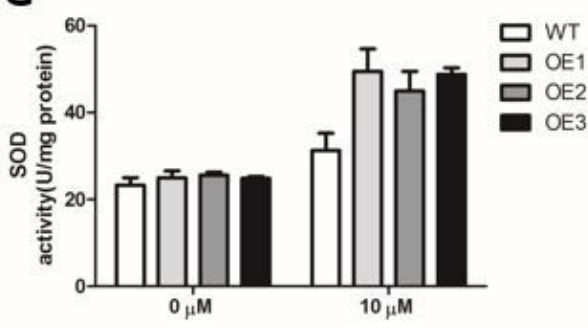

B

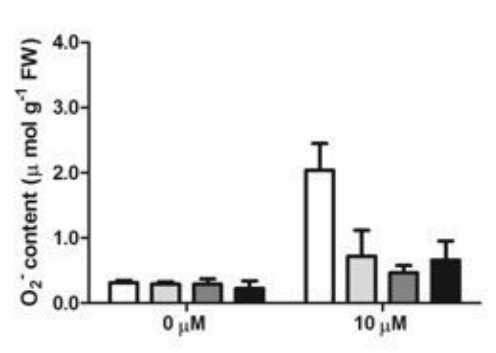

E

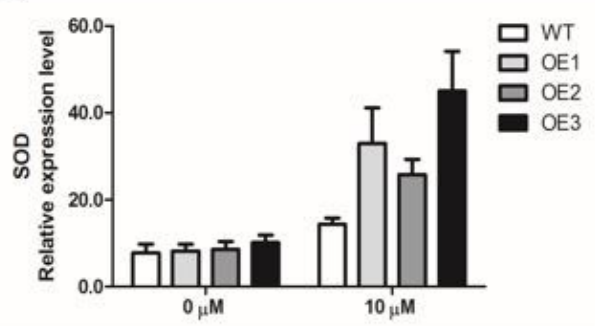

H

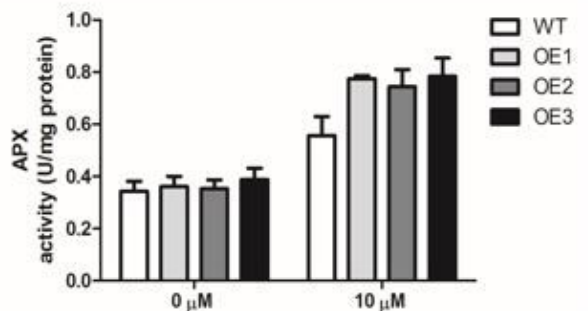

C

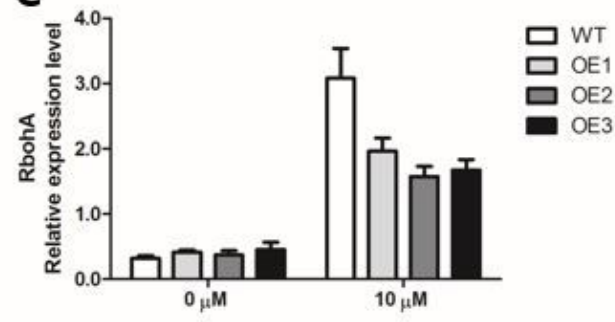

$\mathbf{F}$

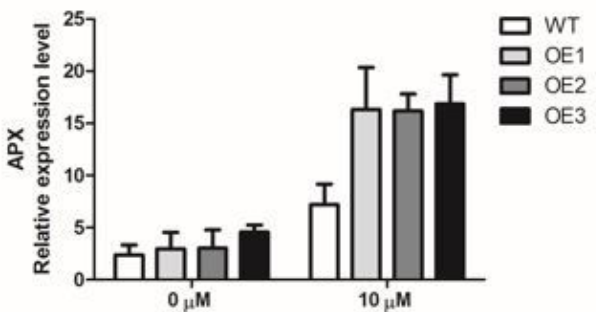

I

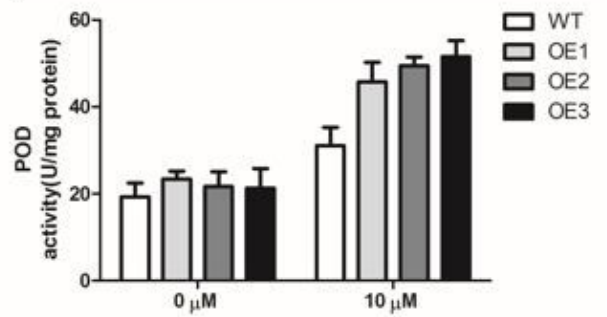

Figure 5

Expression analysis ABA-associated genes in AsTal1 OE lines and WT plants with or without ABA treatment. (A) Expression analysis of the gene involving ABA biosynthesis; (B)-(C) Transcript level of ABA catabolic genes CYP707A1 and CYP707A2. (D)-(E) The abundance of ABA-associated genes. All the data were normalized to the actin gene transcript level. 4-day-old seedings were performed vertically on $1 / 2$ MS medium supplemented with $10 \mu \mathrm{M}$ ABA for 4 days. Mean values obtained from three independent experiments were shown in the histogram. 
A

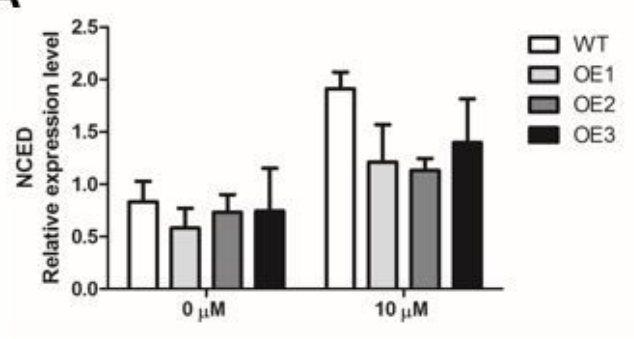

B

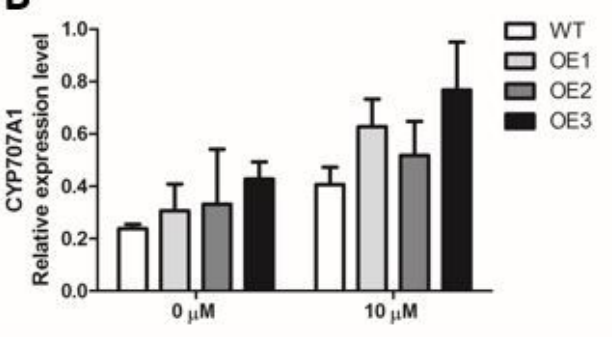

C

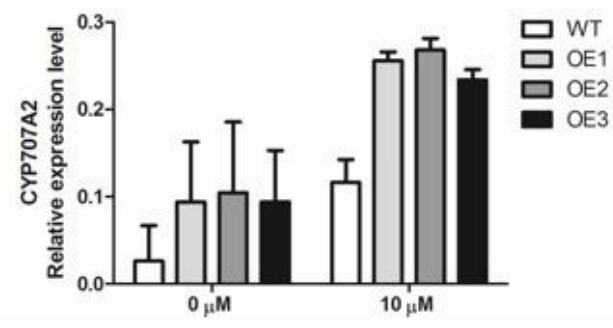

E

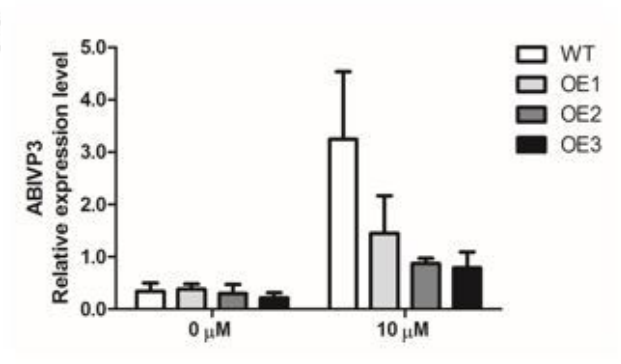

$\mathbf{F}$

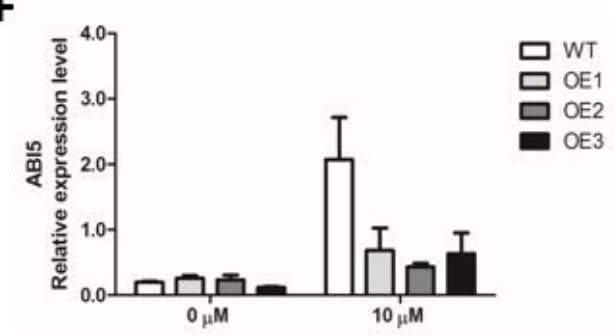

Figure 6

AsTal1 influences the ROS level, and the abundance and activities of ROS responsive enzymes with ABA treatment. (A)-(B) The content of $\mathrm{H} 2 \mathrm{O} 2$ and 02 - in the AsTal1 OE and WT lines. (C)-(D) Expression profiles of NbRbohA and NbRbohB. (E)-(F) Expression analysis of SOD and APX. (G)-(I) Activities of enzyme SOD, APX and POD. 4-day-old seedings were performed vertically on 1/2 MS medium supplemented with 10 $\mu \mathrm{M}$ ABA for 3 days. Error bars represent in the standard deviation of three biological replicates.

\section{Supplementary Files}

This is a list of supplementary files associated with this preprint. Click to download.

- supplementaryfile.docx 